\title{
Avaliação sensório-motora do tornozelo e pé entre idosos diabéticos e não diabéticos
}

\author{
Sensory-motor assessment of ankle and foot among diabetic and non-diabetic elderly
}

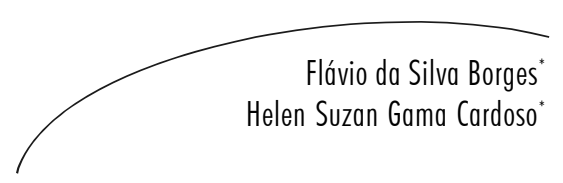

\section{Resumo}

Objetivo: Comparar a força muscular e a sensibilidade do tornozelo e pé entre idosos diabéticos tipo 2 e não diabéticos. Metodologia: Foi realizado estudo transversal, com amostra de 30 idosos, de ambos os sexos, sendo 15 indivíduos diabéticos tipo 2 e não diabéticos. Estes foram selecionados através de prontuários e avaliados por meio de teste de sensibilidade tátil com monofilamento tipo Semmes-Weinstein e teste de força muscular, sendo testados flexores e extensores dos pododáctilos, lumbricais, interósseos, tibial anterior e tríceps sural. Resultados: Verificou-se que não houve diferença na força muscular entre os grupos, possivelmente devido ao programa fisioterapêutico realizado pelo grupo de diabéticos neuropatas, mas em relação à sensibilidade, houve diferença estatística significativa, demonstrando a perda decorrente da neuropatia diabética. Conclusão: Demonstrou-se a perda de sensibilidade tátil no tornozelo e pé dos idosos diabéticos e a fisioterapia pode ter contribuído para o ganho ou a manutenção da força muscular do tornozelo e pé dos idosos diabéticos.

\section{Abstract}

Objective: To compare the muscular strength and sensitivity of the ankle and foot among type 2 diabetic and non-diabetic elderly individuals. Methodology: a crosssectional study was conducted, with a sample of 30 elderly of both genders and 15 individuals with type 2 diabetes and non-diabetic ones. These were selected through records and evaluated through the test of tactile sensitivity with SemmesWeinstein monofilament type, testing flexor, extensor, lumbricals and interosseuos fingers muscle strength, and tibialis anterior and triceps sural. Results: No difference was found in muscle strength between the groups, possibly due to the physiotherapeutic program performed by the group of diabetic neuropathy, but in relation to sensitivity, difference was statistically significant, showing the loss caused by diabetic neuropathy. Conclusion: The loss of tactile sensitivity in the ankle and foot of diabetic elderly was evident and physical therapy may have contributed to their gain or maintenance of muscular strength of ankle and foot.
Palavras-chave: Força

Muscular. Tato. Tornozelo. Pé. Diabetes Mellitus Tipo 2. Fisioterapia (Especialidade).
Key words: Descriptors: Muscle Strength. Touch. Ankle. Foot. Diabetes Mellitus, Type 2. Physical Therapy (Specialty). 


\section{INTRODUÇÃO}

A diabetes mellitus (DM) tipo 2, conhecida como síndrome metabólica, representa cerca de 90\% dos casos, ocorrendo na maior parte em pessoas obesas. ${ }^{1}$ É caracterizada pela diminuição da secreção de insulina e/ou da sensibilidade tecidual a esse hormônio. ${ }^{2}$

O pé diabético é o conjunto das alterações sensório-motoras, vasculares (micro e macrovasculopatia) e biomecânicas que levam à deformidade em decorrência da neuropatia. $\mathrm{O}$ diagnóstico do pé diabético depende do exame clínico adequado, ou seja, uma boa anamnese e exame físico. ${ }^{3}$ É uma complicação crônica que ocorre em média após dez anos de evolução da doença. ${ }^{4}$

Dados epidemiológicos apontam que o pé diabético é responsável por $50 \%$ a $70 \%$ das amputações não traumáticas de membros inferiores, além de concorrer com $50 \%$ das internações hospitalares. ${ }^{4}$

O Ministério da Saúde destacou alguns fatores que aumentam a probabilidade de desenvolver o pé diabético, entre eles o tabagismo, idade superior a 40 anos, diminuição dos pulsos arteriais, hipoestesia, deformidades anatômicas, presença de úlceras e amputações prévias. ${ }^{4}$ Essas causas podem ser justificadas uma vez que, em pessoas idosas, a circulação pode estar diminuída, sobretudo porque os vasos sanguíneos apresentam tendência a arteriosclerose. ${ }^{4}$ A neuropatia diabética (ND) é caracterizada pela diminuição da velocidade de condução nervosa, que está associada a uma diminuição da atividade $d a$ bomba de sódio-potássio no nervo. Essa alteração provoca distúrbios metabólicos e isquêmicos. ${ }^{5}$

Segundo Dullius, ${ }^{4}$ é possível classificar a neuropatia diabética (ND) em somática ou autonômica, focal (incluindo neste tipo as mononeurites e síndromes compressivas) e forma difusa, como as neuropatias proximais, polineuropatias simétricas distais, com acometimento de grandes e pequenas fibras.
Segundo a Sociedade Brasileira de Diabetes, a neuropatia diabética pode ser dividida em: neuropatia sensitiva, que causa perda progressiva da sensibilidade dolorosa, da percepção de pressão, temperatura e propriocepção; neuropatia motora, que acarreta atrofia e enfraquecimento dos músculos intrínsecos do pé, resultando em deformidades e padrão anormal da marcha; neuropatia autonômica, que leva a diminuição ou ausência da secreção sudorípara, ocasionando ressecamento da pele, com presença de rachaduras e fissuras. ${ }^{6}$

Os sinais da polineuropatia sensitiva distal na inspeção são: pele ressecada, veias dilatadas, edema; deformidades de unhas, hálux valgo, articulação de Charcot; atrofia muscular; formação de calos; ulceração plantar; pé seco e quente; pulsos pediosos restritos; perda ou redução dos reflexos tendinosos patelar e aquileu, bem como a perda da dorsiflexão do tornozelo.?

A sensibilidade dos pés e tornozelos pode ser avaliada por diapasão $128 \mathrm{~Hz}$, texturas diferentes e objetos de temperaturas diferentes. No entanto, para a análise da sensibilidade dos pés neste estudo, foi utilizado o teste com monofilamento Semmmes Weinstein, por sua eficácia comprovada em outros estudos e simplicidade de aplicação. ${ }^{8}$

O objetivo fisioterapêutico primário para tratar a DM tipo 2 visa a manter a qualidade de vida do paciente, compreendendo a prevenção de complicações agudas, os sintomas $\mathrm{da}$ hiperglicemia, os efeitos colaterais dos medicamentos, a excessiva morbidade e mortalidade cardiovascular, a retinopatia, a nefropatia e as complicações nos membros inferiores que caracterizam o pé diabético (úlceras e amputações). ${ }^{?}$

É de competência do fisioterapeuta o estudo do movimento humano em todas as suas formas de expressão e potencialidades, quer nas suas alterações patológicas, quer nas suas repercussões psíquicas e orgânicas, com o objetivo de preservar, manter, desenvolver ou restaurar a integridade do órgão, sistema ou função. A preocupação maior é a mobilidade física e como cada patologia pode estar influenciando nas funções do dia a dia dos pacientes. ${ }^{10}$ 
O desaparecimento completo da sensação dolorosa deve ser visto com muito cuidado, pois poderá representar a perda definitiva das terminações nervosas locais, e não uma melhora acentuada do quadro álgico. Existem maneiras simples para se proteger o pé diabético, evitando aparecimento de úlceras e evolução para gangrena e amputação. ${ }^{11} \mathrm{~A}$ maioria dos autores concorda que o controle glicêmico rigoroso é de extrema valia na melhora dos sintomas e na prevenção das complicações ${ }^{12}$.

O objetivo deste estudo foi quantificar as perdas sensório-motoras em tornozelo e pé de idosos com diabetes mellitus tipo 2, em comparação aos idosos não diabéticos.

\section{METODOLOGIA}

Foi realizado estudo transversal nos meses de março a abril de 2008, através da coleta de dados nos prontuários, para o preenchimento da ficha de avaliação elaborada para esta pesquisa, constando: nome, idade, sexo, tempo de diagnóstico, se pratica atividade física e/ou fisioterapia e qual frequência, se tem doenças associadas. Essa ficha foi preenchida no mesmo dia e num único momento com cada voluntário. Em seguida, foi realizado o teste de sensibilidade com monofilamento e avaliação da força muscular do tornozelo e pé.

Este estudo foi aplicado na Instituição de Longa Permanência para Idosos (ILPI) Lar Cecília Ferraz de Andrade - Casa do Vovô, no Distrito Federal, Brasil, e também no Posto de Saúde $n^{\circ}$ 09, localizado no Cruzeiro Novo, Distrito Federal, Brasil, onde há o Projeto pé diabético, com cronograma de atividades às segundas, quartas e sextas-feiras, com um total de 30 idosos.

A amostra experimental foi constituída por 30 idosos acima de 60 anos com idade média de 70 anos. Um grupo era composto por 15 indivíduos com neuropatia diabética de leve a moderada, e o outro por 15 indivíduos do grupo controle, não diabéticos.

Os critérios de inclusão foram: ambos os sexos, voluntários com idade igual ou superior a 60 anos, portadores de diabetes tipo 2 (grupo de diabéticos neuropatas) ou não (grupo controle), ter cognição preservada, que foi atestada por obter pontuação acima de 24 pontos no Mini-Exame do Estado Mental ${ }^{13}$ e sem amputação unilateral ou bilateral dos membros inferiores. Os critérios de exclusão foram: voluntários com doenças neurológicas crônicas, músculo-esqueléticas, psíquicas, vasculares, doenças com alteração da sensibilidade, como a hanseníase.

O teste foi realizado em um ambiente tranquilo e confortável, com o mínimo de interferência externa. O voluntário ficou sentado de frente para a examinadora com o pé apoiado confortavelmente. Primeiro, foi demonstrado o teste para o paciente, utilizando uma área da pele com sensibilidade normal, na mão. ${ }^{11}$

Iniciou-se o teste seguindo a técnica de acordo o Consenso Internacional do pé diabético, com o voluntário de olhos fechados, utilizando um conjunto de monofilamentos de nylon, tipo Semmes-Weinstein (SORRI Bauru'), descrito a seguir. ${ }^{11}$ Foram testados nove pontos na região plantar e um na dorsal. Na região plantar: $1^{\circ}, 3^{\circ}$ e $5^{\circ}$ pododáctilos; $1^{\mathrm{a}}, 3^{\mathrm{a}}$ e $5^{\mathrm{a}}$ cabeças metatarseanas; regiões laterais do meio pé e na região dorsal entre $1^{\circ}$ e $2^{\circ}$ dedos, podendo ser aleatória a sequência de testagem dos pontos. ${ }^{11}$

Iniciou-se o teste com o monofilamento azul $(2,0 \mathrm{~g})$ em todos os pontos descritos acima. Nos pontos em que o paciente não sentiu o filamento azul, prosseguiu-se a avaliação passando para o monofilamento roxo e assim sucessivamente. ${ }^{11}$

O monofilamento é aplicado sobre a pele perpendicularmente, produzindo uma curvatura no fio. Essa curvatura não deve encostar na pele do paciente, para não produzir estímulo extra. A cada ponto testado, o monofilamento azul $(0,2 \mathrm{~g})$ deve ser tocado três vezes seguidas, para garantir que o paciente percebeu o toque. Os demais monofilamentos devem ser tocados apenas uma vez, não causando problemas se forem tocados mais de uma vez. Se o monofilamento escorregar na pele no momento do toque, não considerar a resposta e repetir o teste no mesmo ponto. $\mathrm{O}$ monofilamento deve estar a uma distância de 2 
$\mathrm{cm}$ da área a ser testada e, ao tocar a pele, deve manter a curvatura por 1 ou 1,5 segundo. ${ }^{11}$ Solicitou-se ao paciente que respondesse "sim" quando sentisse o toque.

Cada monofilamento corresponde a um nível funcional representado por uma cor. $\mathrm{Na}$ cor azul $(0,2 \mathrm{~g})$, a sensibilidade tátil está diminuída na mão e normal no pé com dificuldade para discriminar textura (tato leve); na violeta $(2,0 \mathrm{~g})$, a sensibilidade protetora está diminuída tanto na mão quanto no pé, com incapacidade de discriminar textura, formas e temperatura; no vermelho fechado $(4,0 \mathrm{~g})$, há perda de sensibilidade protetora na mão e no pé com incapacidade de discriminação de textura, formas e temperatura; no vermelho com X $(10 \mathrm{~g})$, há perda da sensibilidade protetora no pé, além $\mathrm{da}$ incapacidade de discriminar formas e temperatura; por último, o vermelho aberto $(300 \mathrm{~g})$, no qual permanece apenas sensação de pressão profunda na mão e no pé. ${ }^{14}$

Logo após, foi realizado o teste de função muscular segundo Kendallet al., ${ }^{15}$ correspondendo à escala de $\mathrm{O}$ a 5. De acordo com a força muscular, a escala pode ser graduada através do escore numérico, em ordem crescente: $O$ (Nula: ausência de contração); 1 (Traço: há uma leve contração porém incapaz de produzir movimento); 2 (Fraco: há movimento somente na ausência da gravidade); 3 (Regular: consegue realizar movimento vencendo a gravidade); 4 (Bom: consegue realizar movimento vencendo a gravidade e também uma resistência externa); 5 (Normal: consegue realizar movimento superando uma resistência maior). ${ }^{15}$

Foram dadas instruções gerais aos voluntários, explicando como o exame seria realizado, mostrando a posição em que o membro inferior devia permanecer para realização do movimento. Primeiramente, a avaliadora posicionou a mão palpando a musculatura a ser testada, sendo solicitado ao voluntário que fizesse e mantivesse o movimento. Se o voluntário realizasse o movimento com amplitude total, seria aplicada maior resistência em sentido contrário, mantendo por cinco segundos. Caso o voluntário não realizasse o movimento com amplitude total, não se aplicava mais resistência.
Os testes de função muscular dos interósseos, intrínsecos, flexores e extensores dos dedos e hálux e tibial anterior foram realizados na posição sentada. Logo após, foi solicitado ao paciente que ficasse na posição ortostática, para realização do teste muscular do tríceps sural, cujo teste consistia na elevação dos pododáctilos, empurrando-se o peso do corpo para cima (flexão plantar) sem apoio, sendo repetida por cinco vezes pelo voluntário e correspondendo ao grau $5 .^{15}$

Por último, o voluntário foi orientado a permanecer em pé, subir em uma balança com precisão de 0,1 quilos $(\mathrm{Kg})$ para medir o seu peso e, em seguida, foi verificada a altura com uma fita métrica $(1,50 \mathrm{~m})$ com precisão em milímetros, para se obter o índice de massa corporal (IMC) de cada voluntário.

Todos os voluntários tiveram conhecimento dos procedimentos experimentais pelo pesquisador através do Termo de Consentimento Livre e Esclarecido. Esta pesquisa foi aprovada pelo Comitê de Ética da Universidade Católica de Brasília (Protocolo n 020/2008).

Para análise estatística, foi utilizado o método descritivo, com o teste $t$ para amostras independentes, ou seja, variáveis paramétricas, e o teste de Mann-Whitney na avaliação das variáveis não paramétricas. O software utilizado foi o SPSS 10.0 (Statistical Package for Social Sciences) Windows e o nível de significância adotado foi de $\mathrm{p}$ d" 0,05.

\section{RESULTADOS}

A amostra foi composta por 30 indivíduos, sendo $70 \%$ do sexo feminino, com a média de idade 70,33 $\pm 8,05$ anos. Foi dividida em dois grupos, sendo o grupo diabético composto por 15 indivíduos e o grupo controle composto por 15 indivíduos não diabéticos. Ao comparar as variáveis antropométricas entre os dois grupos, foi realizado um teste $t$ para amostras independentes. A análise demonstrou não haver diferença significativa entre os dois grupos, conforme apresentado na tabela 1 . 
Tabela 1 - Análise descritiva, em média e desvio padrão, da idade, altura, peso, Índice de Massa Corporal (IMC) dos grupos. Brasília, DF, 2008.

\begin{tabular}{lllrc}
\hline & Grupo diabético & Grupo controle & Valor de t & Valor de $\mathrm{p}^{*}$ \\
\hline Idade (anos) & $67,87 \pm 7,17$ & $72,80 \pm 8,36$ & $-1,74$ & 0,09 \\
Altura (m) & $1,58 \pm 0,08$ & $1,63 \pm 0,07$ & $-1,82$ & 0,08 \\
Peso (Kg) & $71,09 \pm 10,08$ & $72,48 \pm 10,99$ & $-0,36$ & 0,72 \\
IMC & $28,59 \pm 4,65$ & $28,47 \pm 4,85$ & 0,07 & 0,94 \\
Tempo DM & $13,00 \pm 4,38$ & - & - & - \\
\hline
\end{tabular}

$\mathrm{m}=$ metro; $\mathrm{Kg}=$ quilograma; IMC = Índice de Massa Corpo-

ral; DM = Diabetes mellitus; * $\mathrm{p} \leq 0,05$

$\mathrm{Na}$ avaliação da força muscular, a análise demonstrou não haver diferença significativa entre os dois grupos. Porém, o grupo GD apresentou menor força muscular nos grupos musculares interósseos, tibial anterior e tríceps sural, conforme descrito na tabela 2.

Tabela 2 - Análise descritiva do teste de força de tornozelo e pé dos grupos. Brasília, DF, 2008.

\begin{tabular}{lllcc}
\hline Teste de força & Grupo diabético & Grupo controle & Valor de t & Valor de $\mathrm{p}^{*}$ \\
\hline Flexor curto do hálux & $4,80 \pm 0,56$ & $4,80 \pm 0,41$ & - & 1,00 \\
Flexor longo do hálux & $4,80 \pm 0,56$ & $4,80 \pm 0,41$ & - & 1,00 \\
Flexor curto dos dedos & $4,80 \pm 0,56$ & $4,80 \pm 0,41$ & - & 1,00 \\
Lumbricais & $4,80 \pm 0,56$ & $4,80 \pm 0,41$ & - & 1,00 \\
Interósseos & $4,53 \pm 0,64$ & $4,73 \pm 0,46$ & $-0,98$ & 0,33 \\
Tibial anterior & $4,40 \pm 0,63$ & $4,67 \pm 0,49$ & $-1,29$ & 0,21 \\
Tríceps Sural & $4,33 \pm 0,62$ & $4,60 \pm 0,51$ & $-1,29$ & 0,21 \\
$* \mathrm{p} \leq 0,05$ & & & &
\end{tabular}

Com relação à avaliação da sensibilidade, por ser uma variável discreta, foi realizado o teste não paramétrico Mann-Whitney. A análise demonstrou haver diferença significativa entre os dois grupos $[\mathrm{Z}=-4,86 ; \mathrm{p}=0,001]$. Podemos observar, conforme apresentado na tabela 3 , que o grupo controle apresenta uma sensibilidade melhor que do grupo diabético. 
Tabela 3 - Análise descritiva do teste de sensibilidade dos grupos. Brasília, DF, 2008.

\begin{tabular}{lcccc}
\hline Monofilamento & Grupo diabético & Grupo controle & $\mathrm{X}^{2}$ & Valor de p \\
\hline Azul $(0,2 \mathrm{~g})$ & - & $20 \%$ & & \\
Violeta $(2,0 \mathrm{~g})$ & - & $27 \%$ & & \\
Vermelho $(4,0 \mathrm{~g})$ & - & $3 \%$ & $-4,86$ & $0,001 \%$ \\
Vermelho com X $(10.0 \mathrm{~g})$ & $37 \%$ & - & & \\
Vermelho magenta $(300 \mathrm{~g})$ & $13 \%$ & - & & \\
Total & $50 \%(15)$ & $50 \%(15)$ & & \\
\hline
\end{tabular}

A tabela 4 mostra que o grupo diabético apresenta dez indivíduos $(66,6 \%)$ que fazem fisioterapia, ao contrário, do grupo não diabéticos que apenas quatro indivíduos $(26,6 \%)$ que estão incluídos no programa de fisioterapia, que é realizada três vezes por semana.

Tabela 4 - Análise descritiva do grupo diabético e do controle que faz fisioterapia. Brasília, DF, 2008.

\begin{tabular}{lll}
\hline & Faz fisioterapia & Não faz fisioterapia \\
\hline Grupo diabético & $66,6 \%(10)$ & $33,4 \%(5)$ \\
Grupo controle & $26,6 \%(4)$ & $73,4 \%(11)$ \\
\hline
\end{tabular}

A figura 1 mostra que no grupo diabético há mais idosos que realizam atividade física, correspondendo a $40 \%$; no entanto, no grupo não diabético apenas $20 \%$ realizam atividade física.

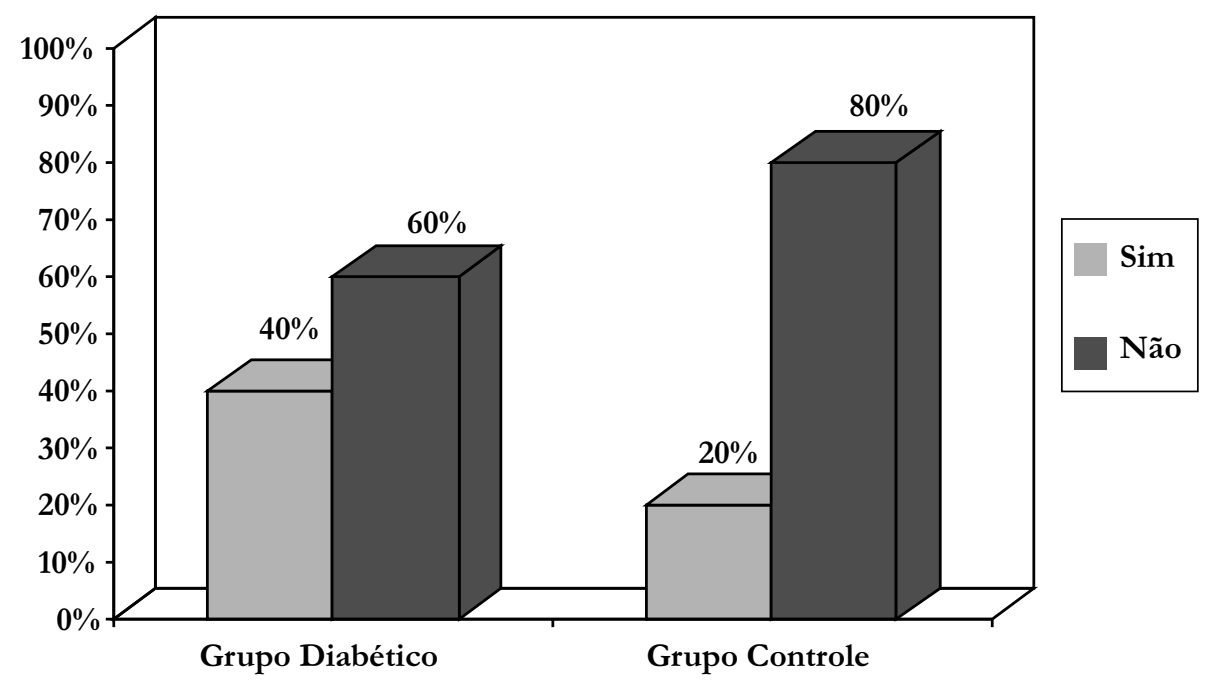

Figura 1: Praticantes de atividade física. 


\section{DISCUSSÃO}

De acordo com a tabela 1, observou se que as médias obtidas entre os grupos são semelhantes, o que torna a amostra homogênea quanto a esses aspectos.

A tabela 2 demonstra a força muscular dos músculos do pé (flexor curto do hálux, flexor longo do hálux, flexor curto dos dedos, lumbricais, interósseos) e da perna (tibial anterior e tríceps sural) de cada grupo, não havendo diferença estatística significativa. Entretanto, os resultados mostram uma leve diminuição da força muscular em ambos os grupos nos músculos interósseos, tibial anterior e tríceps sural.

De acordo com o estudo de Candeloro \& Caromano, ${ }^{16}$ um programa de fisioterapia baseado na hidroterapia, incluindo alongamento, treino de força muscular, mobilização articular, treino de equilíbrio e relaxamento, associado a caminhada, foi eficiente para melhorar a flexibilidade e a força muscular das mulheres idosas com idade entre 65 a 70 anos. Também no estudo de Judge et al., ${ }^{17}$ foi avaliado o efeito de 12 semanas de fisioterapia com treinamento de exercícios posturais, de equilíbrio e força dos músculos extensores do joelho, adutores do quadril, flexores dorsais do pé, extensores do quadril e flexores do joelho, em 16 participantes. O grupo controle, com 15 participantes, realizou exercícios de alongamento geral, sentado em uma cadeira. A idade média dos 31 participantes era de 82,1 anos. Os autores encontraram que o grupo experimental apresentou aumento de força e velocidade do passo. A força dos músculos estudados aumentou em média 32\%. Esses estudos são compatíveis com o achado nesta pesquisa, reforçando que a fisioterapia promove aumento ou manutenção da força muscular nos idosos, o que pode explicar a semelhança de resultados quanto à força muscular entre os grupos.

Em seu estudo, Sacco $^{18}$ encontrou que a função muscular apresentou-se estatisticamente menor no grupo diabético (média de idade de 58 anos) em relação ao grupo controle (média de idade de 53 anos), em todos os grupos musculares testados. No entanto, a população deste estudo não estava incluída em um programa de reabilitação fisioterápica, o que justifica a igualdade da força muscular encontrada nos indivíduos presentes nesta pesquisa.

A maioria dos idosos, tanto do grupo diabético quanto do grupo controle, estava realizando fisioterapia três vezes por semana, com estagiários da Universidade Católica de Brasília orientados por um supervisor docente e fisioterapeuta.

Em relação à tabela 3 , o grupo diabético com neuropatia leve a moderada apresentou a maioria das respostas no monofilamento vermelho com $\mathrm{X}$, correspondendo à perda $\mathrm{da}$ sensibilidade protetora no pé, além da incapacidade de discriminar formas e temperatura. Quanto aos indivíduos não diabéticos, a maioria respondeu ao monofilamento violeta, indicando que a sensibilidade protetora está diminuída no pé, mostrando alteração da sensibilidade nesses indivíduos. Foi encontrado no estudo de Cunha et al., ${ }^{19}$ através da utilização da estesiometria realizada com monofilamento 5,07 (Semmes-Weinstein), que correspondente a $10.0 \mathrm{~g}$, em diabéticos tipo II com média de idade de 65,8 anos, maior perda de sensibilidade do que os diabéticos tipo I. A população diabética se mostrou mais susceptível ao desenvolvimento de úlceras podálicas e ocorrência de neuropatia diabética. ${ }^{19}$

No estudo apresentado por Sacco, ${ }^{18}$ este também encontrou diminuição importante e significativa no grupo dos diabéticos, sendo a maioria das respostas para o monofilamento vermelho $(4,0 \mathrm{~g})$. Não foi o mesmo encontrado neste estudo, porém mostrou que houve diminuição da sensibilidade, conforme encontrado nos resultados desta pesquisa. Esses indivíduos apresentam maior predisposição a sofrer ferimentos e quedas com maior facilidade do que a população do grupo controle. Porém, no grupo controle, encontrou-se diminuição da 
percepção sensitiva, que pode ser explicada pelo tegumento plantar, que, se for muito ou pouco queratinizado, com grande ou pequeno coxim adiposo, ocorre interferência na percepção do estímulo tátil, conforme o resultado encontrado no presente estudo.

A tabela 4 mostra que o grupo diabético apresenta mais indivíduos (10) que fazem fisioterapia do que o grupo dos não diabéticos (4), podendo indicar que, através da fisioterapia, houve a manutenção da força muscular do tornozelo e pé, evitando-se a perda muscular progressiva que leva à dificuldade na deambulação. Matsudo et al., ${ }^{20}$ ao realizarem um programa de treinamento de força de oito semanas com idosos de 86 a 96 anos de idade, com atividades três vezes por semana, obtiveram aumentos de $174 \%$ na força muscular e $48 \%$ na velocidade do passo. No entanto, após quatro semanas sem atividades, observou-se diminuição de $32 \%$ na força, evidenciando a importância da regularidade das atividades físicas. Esse declínio na massa muscular é causado pela redução no tamanho e/ ou pela perda das fibras musculares individuais, ou seja, a sarcopenia. ${ }^{21}$

Conforme a figura 1, ambos os grupos mostram mais indivíduos que não praticam atividade física, porém a maioria realiza fisioterapia três vezes na semana (tabela 4). Isso pode ter compensado o sedentarismo encontrado, pois a atividade fisioterápica regular tem-se mostrado como uma das intervenções mais eficazes na prevenção e tratamento do diabetes e patologias associadas. ${ }^{22}$ Além disso, o exercício aumenta o gasto energético, que combinado com uma ingestão alimentar adequada, pode contribuir para a redução da gordura corporal, tendo em vista que a maioria dos portadores $\mathrm{da}$ diabete tipo 2 apresenta perfil de sobrepeso e obesidade. $^{22}$

Os benefícios do exercício aeróbico realizado tanto na fase aguda, quanto na fase crônica do diabetes, têm sido documentados e incluem a melhoria da sensibilidade à insulina e aumento da captação da glicose sanguínea pelo tecido muscular, bem como das alterações cardiovasculares, que contribuem para um decréscimo dos fatores de risco. ${ }^{22}$

Houve dificuldade em encontrar estudos que utilizaram avaliação semelhante a este estudo, para que fosse possível a comparação dos resultados. Não foi encontrada, na literatura, uma avaliação sensorial e de força muscular com os mesmos parâmetros selecionados no presente estudo e com essa mesma população. Deve-se destacar que há poucas pesquisas em idosos com neuropatia diabética, o que dificulta a comparação dos dados avaliados neste estudo.

Os efeitos da prática da fisioterapia são a manutenção do estado físico do idoso, a melhora da marcha, o controle da evolução de patologias como hipertensão, diabetes e artrose. Essas atividades físicas inserem e aumentam a convivência social e a rede de relações, tornando o idoso mais independente. ${ }^{23}$

\section{CONCLUSÃO}

Com a realização desta pesquisa, foi possível evidenciar que houve diferença na sensibilidade entre idosos diabéticos e não diabéticos, devido à neuropatia diabética. Em relação à força muscular, não houve diferença estatística significativa entre os grupos, pois o grupo diabético fazia fisioterapia regularmente, que provavelmente foi a responsável pelo ganho e manutenção da força muscular do tornozelo e pé.

Este estudo destaca a importância da fisioterapia para a população idosa diabética em relação à saúde e a qualidade de vida. ${ }^{24}$ Cabe salientar que essa prática deve ser sempre orientada por um profissional qualificado, que conheça as características dessa população. ${ }^{25} \mathrm{O}$ fisioterapeuta orienta a realização de exercícios concêntricos e excêntricos com pesos para fortalecimento muscular dos membros inferiores, atividades para equilíbrio e coordenação motora com objetos e o treino funcional da marcha, com o objetivo de ganho de força muscular, equilíbrio e coordenação motora nos MMII. ${ }^{26}$ Podem ser realizados novos 
estudos com esta população, com uma amostra maior e igualmente caracterizada, confirmando o papel da fisioterapia na melhora ou manutenção da força muscular em idosos diabéticos.

\section{REFERÊNCIAS}

1. Albright A, et al. American College of Sports Medicine position stand: exercise and type 2 diabetes. Med Sci Sports Exerc 2000 jul; 32(7): 1354-60.

2. Diniz SF. Influência do diabetes mellitus no processo de reparação de fraturas: estudo experimental em ratos. [dissertação]. Brasília: Programa de Pós-Graduação em Ciências Médicas, Faculdade de Medicina, Universidade de Brasília; 2007.

3. Jablonka $S$, et al. Diabetes mellitus: diagnóstico e tratamento. São Paulo: Fundo Ed. Byk Procienx, 1980. p.171.

4. Karino EM. Identificação de risco para complicações em pés de trabalhadores com diabetes de uma instituição pública da cidade de Londrina-PR. [dissertação]. Ribeirão Preto: Escola de Enfermagem de Ribeirão Preto (EERP); 2004.

5. Dullius J. Diabetes Mellitus, saúde, educação, atividade físicas. Brasília: Editora Universidade de Brasília, FINATEC, 2007.

6. Pedrosa, Hermelinda. Neuropatia diabética periférica. In: Sociedade Brasileira de Diabetes (SBD); 2009. Disponível em: URL: http:// www.diabetesebook.org.br/capitulo/ neuropatia-diabetica-periferica

7. Levin ME, O’Neal FW, Bowker JH, editores. The diabetic foot. 5. ed. St. Louis: 2001.

8. Palmer ML, Epler ME. Fundamentos das técnicas de avaliação musculoesquelética. 2. ed. Rio de Janeiro: Guanabara Koogan, 2000.

9. Schimd H, Neumann C, Brugnara L. O diabetes melito e a desnervação dos membros inferiores: a visão do diabetólogo. Jornal Vascular Brasileiro 2003; 2(1): 37-48.

10. Gagliardi ART. Neuropatia diabética periférica. Jornal Vascular Brasileiro 2003; 2(1): 67-74.

11. Grupo de Trabalho Internacional sobre Pé Diabético. Consenso Internacional sobre o Pé Diabético. Secretaria do Estado da Saúde do Distrito Federal, Brasília, 2001. 100 p.

\section{AGRADECIMENTOS}

A todos que contribuíram para a realização desta pesquisa; às instituições e aos idosos participantes.

12. Kuhn P, et al. O pé diabético. São Paulo: Atheneu, 2006. 249p.

13. Folstein MF, Folstein SE, McHugh PR. "Minimental state": a practical method for grading the cognitive state of patients for the clinician. J Psychiatr Res nov 1975; 12(3): 189-98.

14. Manual de estesiometria (SORRI Bauru), 1990.

15. Kendall FP, McCreary EK, Provance PG. Músculos: provas e funções. 4. ed. São Paulo: Manole, 1995.

16. Candeloro JM, Caromano FA. Efeito de um programa de hidroterapia na flexibilidade e na força muscular de idosas. Rev Bras Fisioter 2007 jul./ago; 11(4): 303-9.

17. Judge JO, et al. Balance improvements in older women: effects of exercise training. Phys Ther 1993 apr; 73(4): 254-62.

18. Sacco ICN, et al. Avaliação das perdas sensório motoras do pé e tornozelo decorrentes da neuropatia diabética. Revista Brasileira de Fisioterapia 2007 jan./fev; 11(1): 27-33.

19. Cunha, et al. Utilização da estesiometria na avaliação da sensibilidade podálica: comparação entre diabetes tipo I e tipo II, 2007. Disponível na URL: www.afb.org.br/ intercobraf/revista/saudepublica/autor/ TATIANA\%20DE\%20SOUSA\%20DA\%20CUNHA\%20\%20825.pdf

20. Matsudo SM, Matsudo VKR, Barros Neto TL. Efeitos benéficos da atividade física na aptidão física e saúde mental durante o processo de envelhecimento. Revista Brasileira de Atividade Física \& Saúde 2000; 5(2): 60-76.

21. Almeida HGG. Diabetes Mellitus: uma abordagem simplificada para profissionais de saúde. São Paulo: Atheneu; 1997.95 p.

22. Fronteira WR, Dawson DM, Slovik DM, organizadores. Exercício físico e reabilitação. Porto Alegre: Artmed, 2001. 420 p.

23. Freitas EV, Cançado F, Py L. Tratado de Geriatria e Gerontologia. Rio de Janeiro: Guanabara Koogan, 2002. 1187 p. 
24. Mazo GZ, Lopes MA, Benedetti TB. Atividade física e o idoso: concepção gerontológica. Porto Alegre: Sulina; 2001. v. 1000, 236 p.

25. Rebelatto JR, Morelli JG, organizadores. Fisioterapia Geriátrica: a prática da assistência ao idoso. São Paulo: Manole, 2004. $455 \mathrm{p}$.

26. Kisner C, Colby LA. Exercícios terapêuticos fundamentos e técnicas. 4. ed. São Paulo: Manole, 2005.841 p.

Recebido: 03/10/2008

Revisado: 27/10/2009

Aprovado: 24/11/2009 